\title{
Evaluation of modified Estimation of Physiologic Ability and Surgical Stress in gastric carcinoma surgery
}

\author{
Yoshio Haga $\cdot$ Yasuo Wada $\cdot$ Hitoshi Takeuchi $\cdot$ \\ Koji Ikejiri $\cdot$ Masakazu Ikenaga $\cdot$ Osamu Kimura
}

Received: 18 January 2011/ Accepted: 17 March 2011/Published online: 3 May 2011

(c) The International Gastric Cancer Association and The Japanese Gastric Cancer Association 2011

\begin{abstract}
Background We recently modified our prediction scoring system "Estimation of Physiologic Ability and Surgical Stress" and have designated the current version mE-PASS. This scoring system has been designed to obtain predicted postoperative mortality rates before surgery and this study was performed to assess its usefulness in elective surgery for gastric carcinoma.
\end{abstract}

\section{Y. Haga $(\bowtie)$}

Institute for Clinical Research, National Hospital Organization

Kumamoto Medical Center, 1-5 Ninomaru,

Kumamoto 860-0008, Japan

e-mail: epass2006@ybb.ne.jp

\section{Y. Haga}

Department of International Medical Cooperation, Graduate School of Medical Sciences Kumamoto University, Kumamoto, Japan

\section{Y. Wada}

Department of Surgery, National Hospital Organization Himeji Medical Center, Himeji, Japan

\section{H. Takeuchi}

Department of Surgery, National Hospital Organization Iwakuni Clinical Center, Iwakuni, Japan

\section{K. Ikejiri}

Department of Surgery, National Hospital Organization Kyushu Medical Center, Fukuoka, Japan

\section{Ikenaga}

Department of Surgery, National Hospital Organization Osaka National Hospital, Osaka, Japan

\section{O. Kimura}

Department of Surgery, National Hospital Organization Yonago Medical Center, Yonago, Japan
Methods We investigated seven variables for mE-PASS and evaluated the postoperative course in 3,449 patients who underwent elective surgery for gastric carcinoma in Japan between August 20, 1987 and April 9, 2007, in order to quantify the predicted in-hospital mortality rates $(R)$. The calibration and discrimination power of $R$ were assessed using the Hosmer-Lemeshow test and the area under the receiver operating characteristic curve (AUC), respectively. The ratios of observed-to-estimated mortality rates (OE ratios) were quantified as a measure of quality. Results The overall postoperative morbidity and mortality rates were 19.0 and $2.0 \%$, respectively. $R$ demonstrated good power in calibration ( $\chi^{2}$ value, 12.5 ; $d f 8 ; P=0.89$ ) as well as discrimination (AUC, $95 \%$ confidence intervals: $0.80,0.75-0.85$ ). The $\mathrm{OE}$ ratios between hospitals ranged from 0.44 to 1.8 . Overall, the $\mathrm{OE}$ ratios seemed to improve with time (OE ratio, 95\% confidence intervals: 1.3, $0.73-2.4$ for the early period between 1987 and 2000; 1.0 , 0.59-1.7 for the middle period between 2001 and 2004; and $0.65,0.36-1.2$ for the late period between 2005 and 2007).

Conclusion Based on these findings, mE-PASS might be useful for medical decision-making and for assessing the quality of care in elective surgery for gastric carcinoma.

Keywords Stomach - Carcinoma - Surgery $\cdot$ Mortality · Prediction

\section{Introduction}

Gastric carcinoma is the second leading cause of death from malignant disease worldwide [1]. Although some chemotherapy regimens have recently been demonstrated to be effective for gastric carcinoma, surgical resection 
with lymph node dissection remains the mainstay of treatment for this tumor. In the 1960s, Japanese surgeons established an extended lymphadenectomy procedure known as D2 [2]. This approach has become a standard procedure in Japan, as it has provided the lowest postoperative morbidity rates accompanied by good prolonged survival rates [3]. General surgeons are taught this technique early during their surgical training. However, two European randomized controlled trials comparing D1 and D2 gastrectomy demonstrated a high surgical mortality exceeding $10 \%$ in the D2 group [4, 5], with no survival benefit for D2 over D1 lymphadenectomy. There were significant problems with these studies, including an association of the high morbidity and mortality rates in the D2 group with inadequate surgical training. D2 gastrectomy has a steep learning curve [6], and may be associated with a higher than expected surgical morbidity and mortality. Recently, several studies demonstrated that D2 lymphadenectomy could be performed with low morbidity and mortality at specialized centers in western countries, as well [7-10] as in developing countries [11-13]. Therefore, in light of these findings, the assessment of surgical quality would be an important issue to evaluate when performing and comparing clinical trials around the world.

We constructed a prediction scoring system for postoperative morbidity and mortality in elective gastrointestinal surgery, designated Estimation of Physiologic Ability and Surgical Stress (E-PSS) [14]. Several cohort studies performed by the authors as well as other researchers have demonstrated reproducible outcomes in predicting adverse postoperative events [14-20]. We recently modified this system and designated this revised version mE-PASS. This modified system estimates postoperative mortality rates before surgery using a reduced number of variables [21]. This study was performed to evaluate the predictive value of mE-PASS in elective surgery for gastric cancer, using data from Japanese regional referral hospitals.

\section{Patients and methods}

\section{Patients}

This study was performed according to the ethical guidelines on epidemiological research jointly developed by the Ministry of Health, Labour and Welfare and the Ministry of Education, Culture, Sports, Science and Technology, Japan. A subset of patients in previous multi-center cohort studies was reanalyzed in the present study [14-17, 21]. Informed consent was obtained from each participant in these prospective studies $[15-17,21]$, but this procedure was waived for retrospective studies [14], because all data were obtained from medical charts and did not include any personal information that could identify any individual patient. For each of the studies, the study design was approved by the relevant ethics review board. Inclusion criteria for this study were patients who underwent elective resection of the stomach for gastric carcinoma between August 20, 1987, and April 9, 2007. Patients who underwent palliative surgery, such as gastrojejunostomy or simple laparotomy, were excluded from this study. These subjects were collected from 50 regional referral hospitals; 44 of these hospitals are associated with the National Hospital Organization. Most of the hospitals are mediumvolume surgical centers, and usually managed approximately 200-800 cases of elective gastrointestinal surgery annually. In total, 3,449 patients met the criteria and were included in this analysis.

Surgical procedures were selected based on tumor location, staging, and histological type, as described in the gastric cancer treatment guidelines in Japan [22]. D2 gastrectomy was a standard procedure for every stage of gastric carcinoma until the late 1990s. Subsequently, modified gastrectomy (D1 + No. 7, 8a or 9 lymphadenectomy) has been preferred for early-stage carcinoma. When endoscopic treatment was impractical or incomplete, local resection of the stomach was usually indicated for differentiated carcinoma that was thought to be confined to the mucosa and measure $2 \mathrm{~cm}$ or less in diameter.

\section{Methods}

We investigated seven variables for mE-PASS and evaluated the postoperative course in 3,449 patients who underwent elective surgery for gastric carcinoma. The parameters of mE-PASS are defined as follows [21].

(1) Preoperative Risk Score (PRS):

$\mathrm{PRS}=-0.0686+0.00345 \quad X_{1}+0.323 \quad X_{2}+0.205$

$X_{3}+0.153 X_{4}+0.148 X_{5}+0.0666 X_{6}$,

where $X_{1}$ is age; $X_{2}$, the presence (1) or absence ( 0 ) of severe heart disease; $X_{3}$, the presence (1) or absence (0) of severe pulmonary disease; $X_{4}$, the presence (1) or absence (0) of diabetes mellitus; $X_{5}$, the performance status index (range 0-4) [23]; and $X_{6}$, the American Society of Anesthesiologists (ASA) physiological status classification (range 1-5) [24].

Severe heart disease is defined as heart failure meeting the criteria of New York Heart Association Class III or IV, or severe arrhythmia requiring mechanical support. Severe pulmonary disease is defined as any condition with a percent vital capacity of less than $60 \%$ and/or a percent forced expiratory volume in $1 \mathrm{~s}$ of less than $50 \%$. Diabetes mellitus is defined according to the World Health Organization criteria [25]. 
(2) Surgical Stress Score fixed (SSSf):

SSSf was determined using the median Surgical Stress Score (SSS) of the original E-PASS in 41 procedures [21]. Values of SSSf in individual procedures are listed in Table 1.

(3) Comprehensive Risk Score fixed (CRSf):

$\mathrm{CRSf}=0.052+0.58(\mathrm{PRS})+0.83(\mathrm{SSSf})$

(4) Predicted in-hospital mortality rates $(R)$ :

CRSf $<0.326 R \fallingdotseq 0$

$\mathrm{CRSf} \geq 0.326 R=-0.0541(\mathrm{CRSf})+0.197(\mathrm{CRSf})^{2}-$ 0.00328

In-hospital mortality and postoperative complications were the primary and secondary endpoints, respectively. The Comprehensive Risk Score fixed (CRSf) and the predicted in-hospital mortality rates $(R)$ were quantified for each patient, using the SSSf for the surgical procedure that was actually performed. Postoperative complications were defined as described previously [26], regarding pneumonia, abdominal abscess, fasciitis, bacteremia, septic shock, septic coagulopathy, anastomotic leak, wound dehiscence, gastrointestinal bleeding, gastrointestinal perforation, pancreatitis, myocardial infarction, cardiogenic shock, cardiopulmonary arrest, stroke, pulmonary embolus, hemoperitoneum, pulmonary failure, renal failure (Grades I and II), wound infection, urinary tract infection, pleural effusion, and hepatic dysfunction. Bacteremia was defined as any condition that included a clinical sign (either fever $\geq 38.5^{\circ} \mathrm{C}$ or shaking chill) and at least one blood culture that was positive for pathogenic organisms, as described previously [26]. Anastomotic stenosis was defined as distinct stenosis that caused a delay in the resumption of solid food intake until after postoperative day 14 . Ileus was defined as obstruction of the small intestines that required long tube decompression or reoperation. Some of these complications were grouped together as follows: cardiac complications including myocardial infarction, cardiogenic shock, and cardiopulmonary arrest; respiratory complications including pneumonia, pulmonary embolus, pulmonary failure, and pleural effusion; and general septic complications including bacteremia, septic shock, and septic coagulopathy.

The severity of postoperative complications was classified using the morbidity score (MS) as described previously [14]; grade 0 , no complication; grade 1, mild complications that are not life-threatening, such as wound infection; grade 2, moderate complications that are potentially life-threatening unless adequate treatment is performed, including pneumonia, anastomotic leakage, and ileus; grade 3, severe organ dysfunction that usually requires mechanical support, being equivalent to stage III in our own classification of organ dysfunction, but precise definitions were determined in seven organs [27]; and grade 4, in-hospital death due to complications.
Statistical analysis

All statistical analyses were performed using the SPSS 17.0 (SPSS, Chicago, IL, USA) software program. Categorical variables were compared between groups using the $\chi^{2}$ test with Yates correction for continuity or, where appropriate, Fisher's exact test. A two-tailed $P$ value of less than 0.05 was considered significant.

The discriminatory power of a model to discriminate patients who died during hospitalization from those who did not was assessed by calculating the area under the receiver operating characteristic curve (AUC) [28]. The AUC ranged from 0.5 to 1.0 and the greater the AUC, the better the model. An AUC of 1.0 indicates a perfect model that shows $100 \%$ sensitivity and $100 \%$ specificity. In contrast, an AUC of 0.5 indicates a completely ineffective model unable to differentiate between real cases and non-cases. Calibration power was assessed by goodness-of-fit testing using the Hosmer-Lemeshow test [29]. This test divides cases into 10 groups in order of predicted probabilities and computes a $\chi^{2}$ statistic from observed and expected frequencies [29]. It tests the null hypothesis that there is no difference between the observed and predicted values of the response variable. Therefore, when the test is not significant, we cannot reject the null hypothesis and say that the model fits the data well. In other words, a good model is indicated by a high $P$ value.

The ratio of observed-to-estimated in-hospital mortality rates (OE ratio) was used as a measure of quality. The $95 \%$ confidence intervals (CIs) of the OE ratio were determined by the method of Katz et al. for risk ratio [30]. When the OE ratio of a hospital is smaller than 1 , the observedmortality rate is lower than the expected rate, indicating that the quality of surgical performance is better than expected. In contrast, an $\mathrm{OE}$ ratio greater than 1 indicates a poorer performance.

\section{Results}

Table 2 shows the demographic data of the subjects. Most patients were old, showing a median age of 69 years, with the oldest patient being 97 years old. The most frequently performed procedure was open distal gastrectomy. Laparoscopic distal gastrectomy was performed for more than $10 \%$ of the distal gastrectomy group.

Table 3 lists the incidences of postoperative complications occurring in this series. Among the 3,449 surgically resected patients, 782 complications occurred in 656 patients $(19.0 \%)$. The most frequent complication was abdominal abscess and the second most frequent was anastomotic leak. Among these 3,449 patients overall, 
Table 1 Surgical Stress Score fixed (SSSf)

\begin{tabular}{|c|c|c|}
\hline Main procedures & Additive procedures & SSSf \\
\hline \multicolumn{3}{|c|}{ Esophagectomy for malignant tumor with reconstruction using gastrointestinal tract } \\
\hline \multicolumn{2}{|l|}{1 via neck, thoracic, and abdominal incision } & 0.824 \\
\hline \multicolumn{2}{|l|}{2 via thoracic and abdominal incision } & 0.693 \\
\hline \multicolumn{2}{|l|}{3 via abdominal incision } & 0.443 \\
\hline \multicolumn{3}{|l|}{ Partial gastrectomy including laparoscopic procedures } \\
\hline \multicolumn{2}{|l|}{1 simple resection } & 0.110 \\
\hline 2 resection for malignant tumor & With or without cholecystectomy & 0.212 \\
\hline \multicolumn{3}{|l|}{ Total gastrectomy including laparoscopic procedures } \\
\hline 1 resection for malignant tumor & With or without cholecystectomy & 0.328 \\
\hline 1 resection for malignant tumor & With splenectomy & 0.352 \\
\hline 1 resection for malignant tumor & With distal pancreatectomy & 0.461 \\
\hline Open choledochotomy with stone removal & With or without cholecystectomy & 0.159 \\
\hline Laparoscopic choledochotomy with stone removal & With or without cholecystectomy & -0.176 \\
\hline \multicolumn{2}{|l|}{ Open cholecystectomy } & 0.129 \\
\hline \multicolumn{2}{|l|}{ Laparoscopic cholecystectomy } & -0.28 \\
\hline \multicolumn{2}{|l|}{ Reconstruction or resection of common bile duct } & 0.208 \\
\hline \multicolumn{2}{|l|}{ Cholecystectomy for malignant tumor } & 0.309 \\
\hline \multicolumn{2}{|l|}{ Resection of common bile duct for malignant tumor } & 0.401 \\
\hline \multicolumn{3}{|l|}{ Hepatectomy } \\
\hline \multicolumn{2}{|l|}{1 Local resection } & 0.191 \\
\hline \multicolumn{2}{|l|}{2 Segmentectomy } & 0.453 \\
\hline \multicolumn{2}{|l|}{3 Lobectomy } & 0.663 \\
\hline \multicolumn{2}{|l|}{4 Extended lobectomy } & 1.025 \\
\hline \multicolumn{2}{|l|}{ Ablation therapy for malignant liver tumor } & 0.117 \\
\hline \multicolumn{3}{|l|}{ Distal pancreatectomy for tumors } \\
\hline \multicolumn{2}{|l|}{1 Resection of pancreatic tail } & 0.277 \\
\hline \multicolumn{2}{|l|}{2 Tumor resection with nodal dissection } & 0.276 \\
\hline \multicolumn{3}{|l|}{ Resection of pancreatic head tumor } \\
\hline \multicolumn{2}{|l|}{1 Pancreaticoduodenectomy } & 0.496 \\
\hline \multicolumn{2}{|l|}{$\begin{array}{l}2 \text { Tumor resection with nodal dissection or resection of pancreatic head } \\
\text { with duodenum preservation }\end{array}$} & 0.612 \\
\hline $\begin{array}{l}3 \text { Tumor resection with surrounding organs such as stomach, colon, } \\
\text { kidney, and adrenal gland }\end{array}$ & & 1.028 \\
\hline 4 Tumor resection with blood vessel reconstruction & & 1.028 \\
\hline Open splenectomy & & 0.0932 \\
\hline Laparoscopic splenectomy & & -0.220 \\
\hline Open adhesiolysis of intestines & & 0.0699 \\
\hline Laparoscopic adhesiolysis of intestines & & -0.260 \\
\hline Resection of small intestines & & \\
\hline 1 Excluding resection for malignant tumor & & 0.1560 \\
\hline Open colectomy & & \\
\hline 1 Resection of small range ${ }^{a}$ & & 0.1448 \\
\hline 2 Total or subtotal resection including laparoscopic procedures & With or without cholecystectomy & 0.1448 \\
\hline 3 Resection for malignant tumors including laparoscopic procedures & With or without cholecystectomy & 0.1448 \\
\hline Laparoscopic colectomy & & -0.220 \\
\hline Rectal resection or amputation & & \\
\hline 1 Resection including laparoscopic procedures & & 0.1951 \\
\hline 2 Low anterior resection including laparoscopic procedures & With or without cholecystectomy & 0.232 \\
\hline 2 Low anterior resection including laparoscopic procedures & With hysterectomy and bilateral oophorectomy & 0.507 \\
\hline
\end{tabular}


Table 1 continued

\begin{tabular}{|c|c|c|}
\hline Main procedures & Additive procedures & SSSf \\
\hline 3 Super low anterior resection or amputation & & 0.410 \\
\hline 3 Super low anterior resection or amputation & With hysterectomy and bilateral oophorectomy & 0.642 \\
\hline 3 Super low anterior resection or amputation & $\begin{array}{l}\text { With resection of urinary bladder followed by } \\
\text { reconstruction using intestines }\end{array}$ & 0.733 \\
\hline
\end{tabular}

SSSf in each procedure was defined as the median value of the Surgical Stress Score (SSS) of the original Estimation of Physiologic Ability and Surgical Stress (E-PASS) scoring system from the previously accumulated data. The original SSS was determined using three intraoperative variables-blood loss, surgical duration, and extent of skin incision (See Refs. [14, 21])

${ }^{a}$ Resection of small range indicates limited resection without nodal dissection

Table 2 Demographic data of the subjects $(N=3,449)$

\begin{tabular}{ll}
\hline Age, years (median, range) & $69,18-97$ \\
Gender (\% male) & $67 \%$ \\
Histological type & $N$ \\
Carcinomas & 3,412 \\
Malignant lymphoma & 27 \\
Gastrointestinal stromal tumor & 10 \\
Surgical procedures & $N$ \\
Total resection of gastric tube via right thoracotomy and & 2 \\
laparotomy & \\
Esophago-gastric resection via left thoracotomy and & 7 \\
laparotomy & \\
Open local resection of stomach & 43 \\
Laparoscopic local resection of stomach & 17 \\
Open distal gastrectomy & 1,916 \\
Laparoscopic distal gastrectomy & 229 \\
Open total gastrectomy & 880 \\
Open total gastrectomy with splenectomy & 146 \\
Open total gastrectomy with distal pancreatectomy & 66 \\
Laparoscopic total gastrectomy & 24 \\
Open proximal gastrectomy & 99 \\
Laparoscopic proximal gastrectomy & 8 \\
Pancreaticoduodenectomy & 10 \\
Pancreaticoduodenectomy with resection of surrounding & 2 \\
organs & \\
\hline
\end{tabular}

68 patients $(2.0 \%)$ died of complications during hospitalization.

Figure 1 shows the relationship between the predicted in-hospital mortality rate $(R)$ and the severity of postoperative complications. As $R$ increased, the proportion of more severe complications significantly increased. As for the discrimination power of mE-PASS to detect in-hospital mortality, $R$ demonstrated a good discrimination power of AUC (95\% confidence intervals) at $0.80(0.75-0.85)$ (Fig. 2). Subsequently, we analyzed the goodness of fit of the mE-PASS model using the Hosmer-Lemeshow test and
Table 3 Incidence of postoperative complications

\begin{tabular}{lrl}
\hline Complications & $N$ & Incidence $(\%)$ \\
\hline Abdominal abscess & 212 & 6.2 \\
Respiratory complications & $118^{\mathrm{a}}$ & $3.3^{\mathrm{a}}$ \\
Anastomotic leak & 104 & 3.0 \\
Wound infections & 72 & 2.1 \\
Anastomotic stenosis & 66 & 1.9 \\
Ileus & 29 & 0.84 \\
General septic complications & $28^{\mathrm{a}}$ & $0.82^{\mathrm{a}}$ \\
Pancreatitis & 27 & 0.78 \\
Fasciitis & 20 & 0.58 \\
Wound dehiscence & 20 & 0.58 \\
Hemoperitoneum & 20 & 0.58 \\
Hepatic dysfunction & 17 & 0.49 \\
Renal failure Grade II & 15 & 0.43 \\
Cardiac complications & $9^{\mathrm{a}}$ & $0.26^{\mathrm{a}}$ \\
Gastrointestinal bleeding & 6 & 0.17 \\
Urinary tract infection & 6 & 0.17 \\
Stroke & 6 & 0.17 \\
Gastrointestinal perforation & 4 & 0.12 \\
Renal failure Grade I & 3 & 0.087 \\
\end{tabular}

Among 3,449 patients who underwent elective resection for gastric carcinoma, postoperative complications occurred in 656 patients $(19.0 \%)$

a These groups include several complications in each, as described in the "Patients and methods" section

found that mE-PASS demonstrated a high calibration power ( $\chi^{2}$ value, 12.5 ; $d f 8 ; P=0.89$ ).

We compared quality of care by analyzing the differences in OE ratios between six hospitals in which more than 200 patients were registered (Table 4). The OE ratios ranged from 0.44 to 1.8 . Two hospitals had values less than 1.0, suggesting a better quality than expected. In contrast, three had values of more than 1.0, but none of the values exceeded 2.0.

Finally, we analyzed the historical change in the quality of care for all subjects (Fig. 3). There was a tendency 


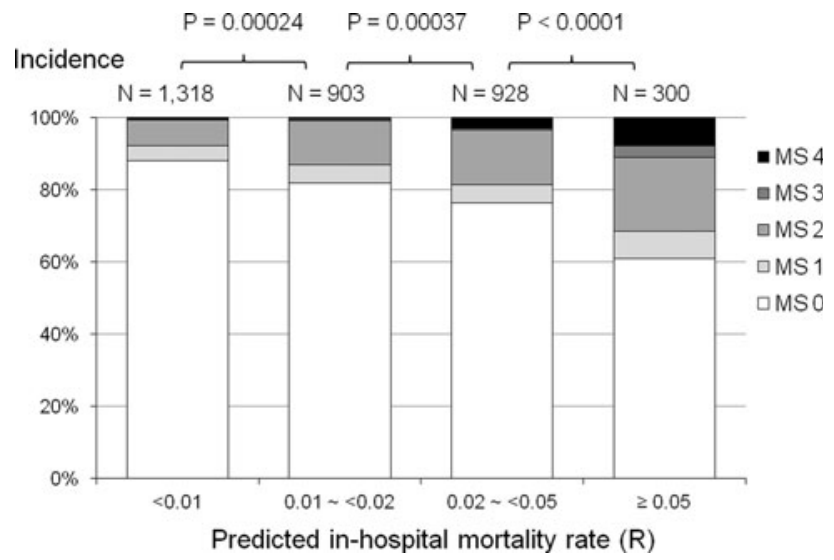

Fig. 1 Relationship between the predicted in-hospital mortality rate $(R)$ and severity of postoperative complications. $M S$ Morbidity score; 0 , no complication; 1 , mild complications; 2 , moderate complications that are potentially life-threatening unless adequate treatment is performed; 3, severe organ dysfunction that usually requires mechanical support; 4 , in-hospital death due to complications

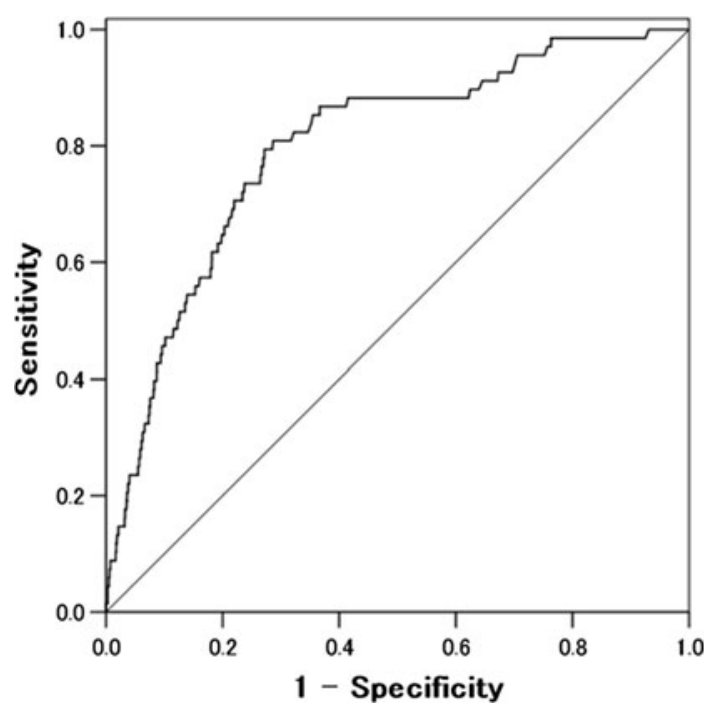

Fig. 2 Receiver operative characteristic (ROC) curve analysis of modified Estimation of Physiologic Ability and Surgical Stress (mE-PASS). ROC curve analysis for mE-PASS was performed to detect in-hospital mortality

towards a reduction in the $\mathrm{OE}$ ratio over time, suggesting that the quality of care has been improved.

\section{Discussion}

This study clearly demonstrated a high discrimination and calibration power of mE-PASS in gastric carcinoma surgery. Although mE-PASS was generated using data from a wide variety of gastrointestinal procedures [21], there has
Table 4 Ratios of observed-to-estimated mortality rates (OE ratios) at individual hospitals

\begin{tabular}{llrrc}
\hline Hospital & $N$ & $\begin{array}{c}\text { Observed } \\
\text { deaths }\end{array}$ & $\begin{array}{c}\text { Estimated } \\
\text { deaths }\end{array}$ & OE ratio (95\% CI) \\
\hline A & 253 & 7 & 6 & $1.0(0.36-2.8)$ \\
B & 242 & 2 & 4 & $0.50(0.092-2.7)$ \\
C & 413 & 4 & 9 & $0.44(0.14-1.4)$ \\
D & 202 & 5 & 4 & $1.3(0.34-4.6)$ \\
E & 397 & 20 & 11 & $1.8(0.88-3.7)$ \\
F & 201 & 6 & 5 & $1.2(0.37-3.9)$ \\
\hline
\end{tabular}

When the OE ratio of a hospital is smaller than 1 , the observed mortality rate of the hospital is lower than the estimated rate, indicating that the quality of surgical performance is better than expected. In contrast, an OE ratio greater than 1 indicates a poorer performance than expected

CI confidence interval

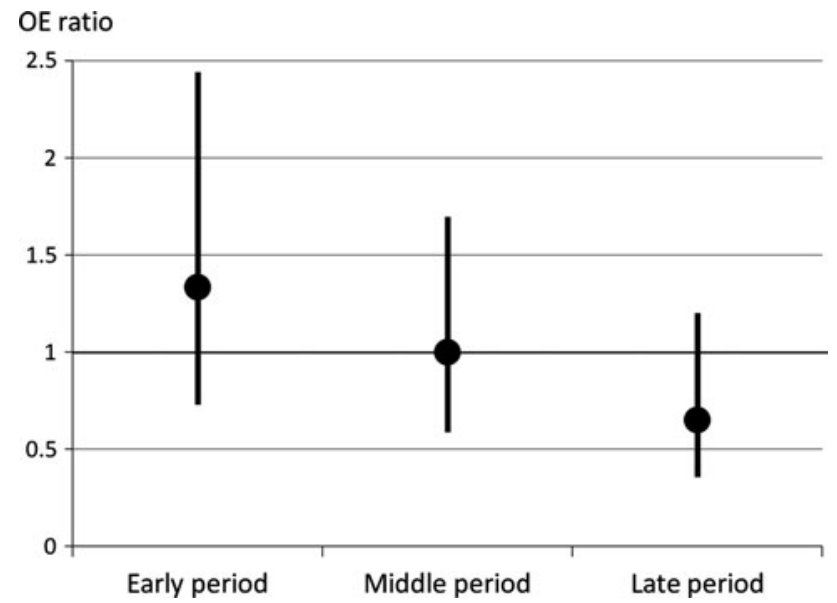

Fig. 3 Historical changes in the quality of care in surgical treatment for gastric carcinoma. The early period was between 1987 and 2000, the middle period was between 2001 and 2004, and the late period was between 2005 and 2007. Dots and bars represent the observed-toestimated mortality rate $(O E)$ ratio and $95 \%$ confidence intervals, respectively

not been any study examining its applicability to gastric surgery. The E-PASS scoring system was constructed on the hypothesis that overwhelming surgical stress exceeding the patient's reserve capacity can lead to the disruption of homeostasis and various kinds of postoperative morbidity. E-PASS has been reported to have a predictive value in other fields of surgery, such as orthopedic surgery [31], vascular surgery [32], and lung surgery [33]. Furthermore, our recent cohort study demonstrated that E-PASS has a high discrimination and calibration power in cardiac surgery as well (unpublished data). Therefore, the above 
hypothesis may be a universal principle for surgical treatment.

Using the original E-PASS scoring system, the predicted mortality rates are obtained after the completion of surgery, because the scoring system requires intraoperative variables, such as blood loss and surgical duration. Therefore, we revised the model in order to obtain a predicted mortality rate preoperatively, and we designated this modified scoring system mE-PASS [21]. This revision reduces the number of variables from ten to seven. Using mE-PASS, surgeons can preoperatively discuss the risk of surgery and the selection of procedures with their patients. For example, an 83-year-old man with severe emphysema, performance status index 1, and ASA class 3 was diagnosed with IIc type early carcinoma in the upper stomach. Preoperative contrast computed tomography (CT) did not demonstrate any evidence of lymph node metastasis. His PRS was 0.77. If he undergoes total gastrectomy, his predicted inhospital mortality rate will be $7.2 \%$. However, if he undergoes local resection, his predicted in-hospital mortality rate will be $3.3 \%$. Of course, the predicted mortality rates represent only average risk and not the attending surgeon's risk. However, we were able to exhibit a difference in risk between the procedures.

Surgical audits comparing hospitals will drive quality improvement. The United States Department of Veterans Affairs (VA) established the National Surgical Quality Improvement Program in all VA hospitals performing surgery in 1994. The inception of these programs resulted in a significant reduction of postoperative morbidity and mortality rates [34]. If we perform such a nation-wide program using mE-PASS in gastric carcinoma surgery, every hospital will try to minimize the OE ratio, leading to significant quality improvement.

The surgical procedure actually performed is sometimes different from that initially scheduled, e.g., the preoperative plan is distal gastrectomy, but total gastrectomy is actually performed. When utilizing mE-PASS to perform a surgical audit, $R$ should be calculated using SSSf for the surgical procedure that was actually performed. Otherwise, hospitals might obtain a lower value of the $\mathrm{OE}$ ratio by basing the calculation on a planned procedure that carried a higher risk than the procedure that was actually performed.

Our study demonstrates quality improvement over time in the overall series. Some of the coagulation devices used for vessel sealing, such as harmonic scissors and the LigaSure $^{\mathrm{TM}}$ (Covidien, Dublin, Ireland), have been utilized since the late 1990s. These devices have enabled the reduction of intraoperative blood loss and shortened the duration of surgery [35]. The recent spread of these devices may contribute to quality improvement over time. Another possible reason for the quality improvement is the application of modified gastrectomy instead of D2 to early carcinoma, which has been recommended in gastric cancer treatment guidelines in Japan since 2001. Furthermore, distal pancreatectomy and splenectomy have not been performed routinely with total gastrectomy, because there have not been significant survival benefits reported but the morbidity rates are higher [36]. These less invasive procedures may reduce postoperative morbidities in high-risk patients.

The formula for mE-PASS was constructed using the data of operations performed between 1987 and 2002. Therefore, overestimation of mE-PASS was observed in the late period of the present study, from 2005 to 2007. The quality of care determined by the OE ratio may be further improved in the future. Therefore, re-calibration of $\mathrm{mE}$ PASS should be considered periodically.

In conclusion, this study demonstrated the predictive value of mE-PASS in gastric carcinoma surgery. This system can be applied to clinical practice, clinical research, and the assessment of quality of care. All of these efforts will contribute to improving the quality of gastric carcinoma surgery in the future.

Acknowledgments This study was supported by a grant for National Hospital Organization Multi-Center Clinical Research for Evidence-Based Medicine.

Conflict of interest The authors declare that there are no conflicts of interest related to the contents of this manuscript.

\section{References}

1. Kamangar F, Dores GM, Anderson WF. Patterns of cancer incidence, mortality, and prevalence across five continents: defining priorities to reduce cancer disparities in different geographic regions of the world. J Clin Oncol. 2006;24:2137-50.

2. Kajitani T. The general rules for the gastric cancer study in surgery and pathology: part 1-clinical classification. Jpn J Surg. 1981;11:127-39.

3. Sasako M, McCulloch P, Kinoshita T, Maruyama K. New method to evaluate the therapeutic value of lymph node dissection for gastric cancer. Br J Surg. 1995;82:346-51.

4. Bonenkamp JJ, Songun I, Hermans J, Sasako M, Welvaart K, Plukker JT, et al. Randomised comparison of morbidity after D1 and D2 dissection for gastric cancer in 996 Dutch patients. Lancet. 1995;345:745-8.

5. Cuschieri A, Fayers P, Fielding J, Craven J, Bancewicz J, Joypaul $\mathrm{V}$, et al. Postoperative morbidity and mortality after D1 and D2 resections for gastric cancer: preliminary results of the MRC randomised controlled surgical trial. The Surgical Cooperative Group. Lancet. 1996;347:995-9.

6. Parikh D, Johnson M, Chagla L, Lowe D, McCulloch P. D2 gastrectomy: lessons from a prospective audit of the learning curve. Br J Surg. 1996;83:1595-9.

7. Degiuli M, Sasako M, Calgaro M, Garino M, Rebecchi F, Mineccia M, et al. Morbidity and mortality after D1 and D2 gastrectomy for cancer: Interim analysis of the Italian Gastric Cancer Study Group (IGCSG) randomised surgical trial. Eur J Surg Oncol. 2004;30:303-8. 
8. Lamb P, Sivashanmugam T, White M, Irving M, Wayman J, Raimes S. Gastric cancer surgery - a balance of risk and radicality. Ann R Coll Surg Engl. 2008;90:235-42.

9. Degiuli M, Sasako M, Ponti A, Italian Gastric Cancer Study Group, et al. Morbidity and mortality in the Italian Gastric Cancer Study Group randomized clinical trial of D1 versus D2 resection for gastric cancer. Br J Surg. 2010;97:643-9.

10. Baiocchi GL, Tiberio GA, Minicozzi AM, Morgagni P, Marrelli $\mathrm{D}$, Bruno L, et al. A multicentric Western analysis of prognostic factors in advanced, node-negative gastric cancer patients. Ann Surg. 2010;252:70-3.

11. Zilberstein B, da Costa Martins B, Jacob CE, Bresciani C, Lopasso FP, de Cleva R, et al. Complications of gastrectomy with lymphadenectomy in gastric cancer. Gastric Cancer. 2004;7:254-9.

12. Shrikhande SV, Shukla PJ, Qureshi S, Siddachari R, Upasani V, Ramadwar M, et al. D2 lymphadenectomy for gastric cancer in Tata Memorial Hospital: Indian data can now be incorporated in future international trials. Dig Surg. 2006;23:192-7.

13. Portanova M, Vargas F, Lombardi E, Mena V, Carbajal R, Palacios N, et al. Results of specialization in the surgical treatment of gastric cancer in Peru. Gastric Cancer. 2007;10:92-7.

14. Haga Y, Ikei S, Ogawa M. Estimation of Physiologic Ability and Surgical Stress (E-PASS) as a new prediction scoring system for postoperative morbidity and mortality following GI surgery. Surg Today. 1999;29:219-25.

15. Haga Y, Ikei S, Wada Y, Takeuchi H, Sameshima H, Kimura O, et al. Evaluation of an Estimation of Physiologic Ability and Surgical Stress (E-PASS) scoring system to predict postoperative risk: a multicenter prospective study. Surg Today. 2001;31: 569-74.

16. Haga Y, Wada Y, Takeuchi H, Sameshima H, Kimura O, Furuya T. Estimation of surgical costs using a prediction scoring system estimation of physiologic ability and surgical stress. Arch Surg. 2002;137:481-5.

17. Haga Y, Wada Y, Takeuchi H, Kimura O, Furuya T, Sameshima $\mathrm{H}$, et al. Estimation of physiologic ability and surgical stress (E-PASS) for a surgical audit in elective digestive surgery. Surgery. 2004;135:586-94.

18. Oka Y, Nishijima J, Oku K, Azuma T, Inada K, Miyazaki S, et al. Usefulness of an estimation of physiologic ability and surgical stress (E-PASS) scoring system to predict the incidence of postoperative complications in gastrointestinal surgery. World $\mathbf{J}$ Surg. 2005;29:1029-33.

19. Banz VM, Studer P, Inderbitzin D, Candinas D. Validation of the estimation of physiologic ability and surgical stress (E-PASS) score in liver surgery. World J Surg. 2009;33:1259-65.

20. Hashimoto D, Takamori H, Sakamoto Y, Ikuta Y, Nakahara O, Furuhashi $\mathrm{S}$, et al. Is an estimation of physiologic ability and surgical stress able to predict operative morbidity after pancreaticoduodenectomy? J Hepatobiliary Pancreat Sci. 2010;17:132-8.

21. Haga Y, Ikejiri K, Wada Y, Takahashi T, Ikenaga M, Akiyama N, et al. A multicenter prospective study of surgical audit systems. Ann Surg. 2011;253:194-201.
22. Nakajima T. Gastric cancer treatment guidelines in Japan. Gastric Cancer. 2002;5:1-5.

23. Oken MM, Creech RH, Tormey DC, Horton J, Davis TE, McFadden ET et al. Toxicity and response criteria of the Eastern Cooperative Oncology Group. Am J Clin Oncol. 1982;5:649-55.

24. Owens WD, Felts JA, Spitznagel EL. ASA physiological status classifications: a study of consistency of ratings. Anesthesiology. 1978;49:239-43.

25. Alberti KG, Zimmet PZ. Definition, diagnosis and classification of diabetes mellitus and its complications. Part 1: diagnosis and classification of diabetes mellitus provisional report of a WHO consultation. Diabet Med. 1998;15:539-53.

26. Doglietto GB, Gallitelli L, Pacelli F, Bellantone R, Malerba M, Sgadari A, et al. Protein-sparing therapy after major abdominal surgery: lack of clinical effects. Ann Surg. 1996;223:357-62.

27. Haga Y, Beppu T, Doi K, Nozawa F, Mugita N, Ikei S, et al. Systemic inflammatory response syndrome (SIRS) and organ dysfunction following gastrointestinal surgery. Crit Care Med. 1997;25:1994-2000.

28. Akobeng AK. Understanding diagnostic tests 3: receiver operating characteristic curves. Acta Paediatrica. 2007;96:644-7.

29. Hosmer DW, Lemeshow S. Summary measures of goodness-offit. In: Applied logistic regression. 2nd ed. New York: Wiley; 2000. p. 144-67.

30. Katz D, Baptista J, Azen SP, Pike MC. Obtaining confidence intervals for the risk ratio in cohort studies. Biometrics. 1978;34:469-74.

31. Hirose J, Mizuta H, Ide J, Nakamura E, Takada K. E-PASS for predicting postoperative risk with hip fracture: a multicenter study. Clin Orthop Relat Res. 2008;466:2833-41.

32. Tang T, Walsh SR, Fanshawe TR, Gillard JH, Sadat U, Varty K, et al. Estimation of physiologic ability and surgical stress (E-PASS) as a predictor of immediate outcome after elective abdominal aortic aneurysm surgery. Am J Surg. 2007;194: 176-82.

33. Yamashita S, Haga Y, Nemoto E, Nagai S, Ohta M. E-PASS (the estimation of physiologic ability and surgical stress) scoring system helps the prediction of postoperative morbidity and mortality in thoracic surgery. Eur Surg Res. 2004;36:249-55.

34. Khuri SF, Daley J, Henderson W, Hur K, Demakis J, Aust JB, et al. The Department of Veterans Affairs' NSQIP: the first national, validated, outcome-based, risk-adjusted, and peer-controlled program for the measurement and enhancement of the quality of surgical care. National VA Surgical Quality Improvement Program. Ann Surg. 1998;228:491-507.

35. Macario A, Dexter F, Sypal J, Cosgriff N, Heniford BT. Operative time and other outcomes of the electrothermal bipolar vessel sealing system (LigaSure) versus other methods for surgical hemostasis: a meta-analysis. Surg Innov. 2008;15:284-91.

36. Kitamura K, Nishida S, Ichikawa D, Taniguchi H, Hagiwara A, Yamaguchi $\mathrm{T}$, et al. No survival benefit from combined pancreaticosplenectomy and total gastrectomy for gastric cancer. Br J Surg. 1999;86:119-22. 